\title{
Possible studies on adhesion in space
}

\author{
G VENKATARAMAN \\ Reactor Research Centre, Kalpakkam 603 102, India \\ MS received 17 June 1980
}

\begin{abstract}
This paper reviews possible experiments on adhesion thatca n be carried out in a space lab environment. After introducing the basic concepts of adhesion physics, the proposed European experiment on surface energy measurement by dy namic methods in space environment is discussed and reviewed. It is concluded that as of now morc studies on adhesion in terrestrial environment are desirable than space experiments.
\end{abstract}

Keywords. Adhesion; surfacc energy; space experiments.

"There are agents in Nature able to make particles of joint stick together by very strong attractions, and it is the busirtess of experimental philosophy to find them out"

- Newton

\section{Introdaction}

This paper will deal with possible studies pertaining to adhesion which can be carried out in Spacelab-like environment. Our concern will be mainly with the basic aspects of the phenomenon of adhesion, and related questions pertaining to surface physics. However, it may be pertinent to begin with a brief discussion of how adhesives are used in space technology. This will provide, though perhaps tenuously, some justification for engaging in basic studies !

\section{Adhesives in space technology}

The ASTM Bulletin defines adhesion as "the state in which two surfaces are held together by interfacial forces". An adhesive is a material that binds two surfaces by surface attachment.

For many years, adhesives had limited use in aircraft and spacecraft. The Apollo spacecraft was the first large airborne vehicle in which adhesives were utilised throughout most of the load-bearing structure. In the initial stages, there were many problems. Most of the bonds had to be scrapped or reworked due to voids caused by volatile matter escaping during the cure of adhesives. This led to a programme for developing stable, high-temperature adhesives, free from volatile formation.

The basic strategy was to try and introduce a stabilising linkage in the main chain of the epoxy monomer (Spiker and Barnes 1970). The chemical group 
selected for this purpose was carborane $\mathrm{C}_{2} \mathrm{~B}_{10} \mathrm{H}_{12}$. The carbon and the boron atoms in carborane are arranged in an isocahedral array, and three isomers, ortho, meta and para, are possible, depending on the location of the carbon atoms. In orthocarborane, the two carbon atoms are adjacent to each other, and have a strong electron-withdrawing characteristic. Consequently, when this unit is bonded to other groups, the chemistry of these other groups is strongly affected. The structure of the substituted carborane is represented by

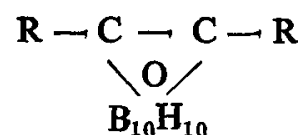

where $R$ belongs to the epoxy. The monomer properties are substantially altered by bonding to carborane, and overall improved stability results. Incidentally we see here basic science in action to solve a practical problem.

\section{Surface energy}

We now address ourselves to the subject proper, and begin with a consideration of a crucial quantity, namely, the specific surface free energy. Denoted by $\gamma$, it is often abbreviated simply as surface energy. It is defined as the isothermal reversible work required for creating $1 \mathrm{~cm}^{2}$ of a new surface. We can, for example, visualise this surface to be produced by applying a tension on a long prism with a square cross-section, and having a $1 \mathrm{~cm}$ edge. Further, the breaking is assumed to be done in a perfect vacuum. Since an energy $\gamma$ is associated with each of the two surfaces created, it is evident by a converse argument that, if the solid could be restored to its original condition by a perfect adhesion of the two surfaces, the energy gained would be $2 \gamma$.

Observe that $\gamma$ is essentially a macroscopic quantity. Though a tensor, we shall treat it here as a scalar. It is a convenient measure of surface properties, and fingerprints the latter the same way the yield stress, for example, gives a comprehensive feel for the mechanical properties of materials.

The calculation of $y$ from first principles has attracted considerable attention especially for atoms interacting via dispersion forces. We remind ourselves that such forces are operative mainly for closed-shell atoms.

The physical origin of these forces is schematically illustrated in figure 1. Here it is assumed that the interaction is instantaneous, i.e., there is no delay in the inducing of a dipole moment on atom 2 due to a fluctuation in atom 1. Clearly this is not valid, especially for large $r$, since signals cannot travel faster than the velocity of light $c$. Retardation effects thus set in for large separations, and must be allowed for.

The force we have been considering so far is that between isolated atoms. As far as adhesion is concerned, we need to know something about forces between bodies with surfaces. These forces can be calculated by superposing the forces between all distinct pairs of atoms, one each in the two bodies. Table 1 (Israelachivili and Tabor 1972) gives a feel for such forces for different geometrics. 


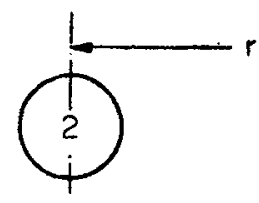

(a) Closed-shell

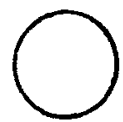

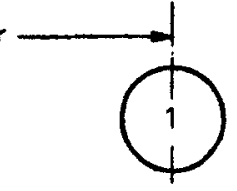

atoms

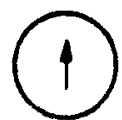

(b) Dipole produced in 1 by flucluotion
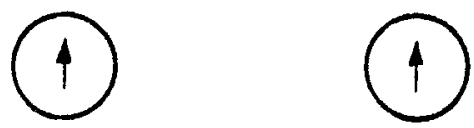

(c) Dipole induced in 2

$$
\begin{aligned}
\text { Interaction energy } & \sim \frac{\mu_{1} \mu_{2}}{r^{3}} \\
& \sim k \frac{e^{2} a_{0}^{2}}{r^{6}}
\end{aligned}
$$

Figure 1. Origin of dispersion forces. (a) depicts two closed shell atoms in their respective ground states. A momentary fluctuation of charge in 1 can lead to an electric dipole moment as in (b). This dipole could then induce another in atom 2 as sketched in (c). The interaction energy goes as $r^{-6}$. The constant is roughly given by $K e a_{0}^{2}$ where $a_{0}$ is the Bohr radius and $K$ the atomic polarisability.

Table 1. Forces of relevance to adhesion.

$$
\quad F=\frac{\text { Const }}{d^{7}} \quad F=\frac{\text { Const }}{d^{8}} \quad F=\frac{\text { Const }}{d^{4}} \quad F=\frac{\text { Const }}{d^{5}}
$$

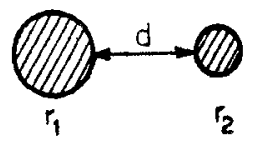


To see the connection with $\gamma$, let us suppose that the atoms interact via the Lennard-Jones (LJ) potential

$$
v(r)=4 \epsilon\left\{(\sigma / r)^{12}-(\sigma / r)^{6}\right\}
$$

where $\epsilon$ and $\sigma$ are constants. The former is related to the potential well depth and the latter to the hard-core radius of the interacting atom. We recall that the Lennard-Jones potential is a phenomenological two-body potential which incorporates, besides the repulsion, an attraction having the same form as that arising from dispersion.

Referring now to figure 2, if $\sigma(z)$ denotes the force between the two surfaces when the separation is $z$, then

$$
\Delta F=-2 \gamma=-\int_{z_{0}}^{\infty} \sigma(z) d z
$$

where $z_{v}$ is the equilibrium separation. For the $\mathbf{L J}$ potential,

$$
z_{0}=(2 / 15)^{1 / 6} \sigma
$$

and $\quad \sigma(z)=\frac{c}{z^{3}}\left[1-\left(z_{0} / z\right)^{6}\right]$

where $\quad c=\frac{1}{3}\left(4 \pi \epsilon \sigma^{6} n^{2}\right)$,

$n$ being the density of the solid. On carrying through the integration, it is found

$$
\gamma=3 c / 16 z_{0}^{2} \text {. }
$$

This exercise, though elementary, gives a feel for how surface energy is calculated from basic considerations. The same technique can be applied to solids with other types of bonding, e.g., ionic, covalent, etc. Table 2 gives some results for ionic crystals (Gregg 1965) which, though outdated, are good enough to convey a feel for the magnitudes involved.

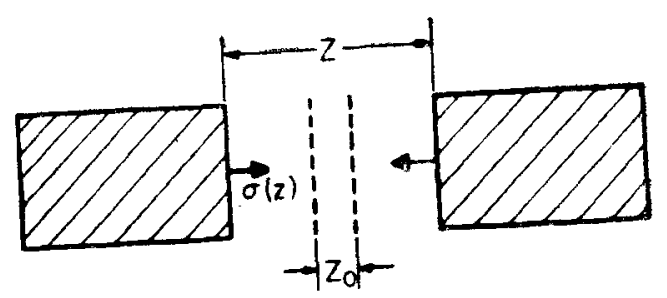

Figure 2. Schematic drawing to illustrate the calculation of $\gamma$. When the separation between the surfaces is $z$, the force of attraction is $\sigma(z)$. $\quad \gamma$ is obtained by calculating the work required to take the two surfaces from their equilibrium separation $Z_{0}$ to infinity. 
Table 2. Surface energies for some ionic crystals.

\begin{tabular}{|c|c|c|c|c|c|c|}
\hline & \multicolumn{2}{|c|}{$\mathrm{NaCl}$} & \multicolumn{2}{|c|}{$\mathrm{NaBr}$} & \multicolumn{2}{|c|}{$\mathrm{KCl}$} \\
\hline Plane & 130 & 110 & 100 & 110 & 100 & 110 \\
\hline$\gamma(\mathrm{calc})$ & 189 & 445 & 177 & 396 & 163 & 352 \\
\hline$\gamma(\operatorname{expt})$ & & & & & & \\
\hline
\end{tabular}

Notes : (1) $\gamma$ is quoted in erg $\mathrm{cm}^{-2}$.

(2) The calculated values are for $0^{\circ} \mathrm{K}$, and reproduced from Gregg (1965).

(3) The experimental value quoted tor $\mathrm{NaCl}$ is derived trom surface tension measurements made above the melting point. This value has been extrapolated to $0^{\circ} \mathrm{K}$. This number also has been derived from Gregg.

For many years, surface forces were calculated only for atoms interacting, via dispersion forces. Lately calculations for other kinds of solids, including metals have started appearing. All these involve complications of their own. For instance, for metals one has to worry about the role played by the electron gas. Similarly, for covalent crystals, one has to reckon with bond-bending forces and so on. An additional complication is that in some cases, the surface layer has a different structure as compared to those beneath; this has been revealed by LEED experiments.

\section{Concerning roughness}

Implicit in the above analysis is the assumption that the surface is smooth, down to the atomic level. In practice this is not the case, and one rather has the situation depicted in figure 3 .

This is a convenient point to digress a bit on the question of roughness. At first sight, it might appear that the roughness of a surface depends merely on handling. It however turns out that this is not always the case. In crystal growth, it is observed that cooperative effects can considerably influence the surface structure. Below a certain temperature $T_{R}$, the surface is generally flat with a few aggregation of atoms and a few vacancies within the plane. Above $T_{R}$, the surface becomes rough.

Figure 4 shows models of crystal surfaces obtained by Monte-Carlo simulation (Muller Krumbhaar 1979). The transition to a rough structure is visible. Referred to as the roughening transition, it has similarities to phase transition.

The roughening of a three-dimensional lattice system is ascribed essentially to fluctuations, the efficacy of the latter being dictated by the energetics involved. Approaching $T_{R}$ from below, only small wavelength fluctuations are initially favoured. Thus, at first, only small islands and holes are formed, surrounded by steps of finite length. Towards $T_{R}$, the free energy of such steps decreases and 
Ideal surfaces:

Clean, Atomically smooth, Planar

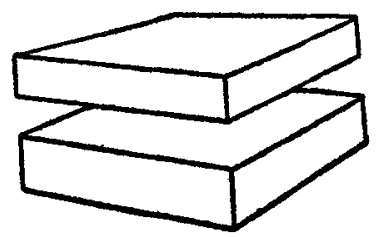

Perfect (complete)

contoct

Strong joint

(a)

Real surfaces:

Ditty, Rough, Nonplanar

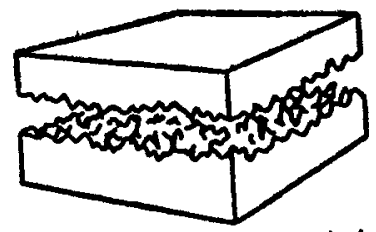

Imperfect (incomplete) contact

Weak joint

(b)

Figure 3. Real life surfaces are seldom smooth as in (a). Roughness is always present, and can influence adhesion.

the surface develops terrace-like structures. Whereas below $T_{R}$, these structures are local, above that temperature their lateral extent diverges resulting in the entire surface being covered with asperites.

A quantitative description of roughening can be given by introducing $h(x)$, the relative height from the lattice plane at the point $x$. The moments $\left\langle[h(x)]^{2 n}\right\rangle$ diverge like $\left(T-T_{R}\right)_{n}$ where $\theta_{n}$ are exponents. The relaxation time $\tau_{2}$ associated with the second-order correlation $\left\langle h\left(x_{1} 0\right) h\left(x_{2}, t_{2}\right)\right\rangle$ also shows a divergence as in figure 5 (Swendsen 1976), which is reminiscent of critical slowing down. As is to be expected, these divergences arise essentially due to the fact the free energy of the surface has a singularity although weak. Owing to the weakness of this singularity, the roughening point cannot be experimentally determined by measuring thermodynamic quantities but certainly it shows up in more subtle quantities.

Let us now consider the effect of roughness on adhesion. For illustration, we envisage the situation in figure 6 and assume the material involved is soft, e.g., indium. When a load is applied, it will clearly act only through the contact areas and as a result, the force in each of these would be much more than what it would otherwise have been, had the surfaces been smooth. In many of these contact regions, the force acting may be sufficient to cause plastic deformation, leading to increased contact in the neighbourhood. In turn, deformation could occur around the new contact regions, promoting further adhesion. In fact, there could be an autozatalytic growth of adhesion, and this is precisely what happens when lumps of wax are pressed together. Despite the low surface energy, and 

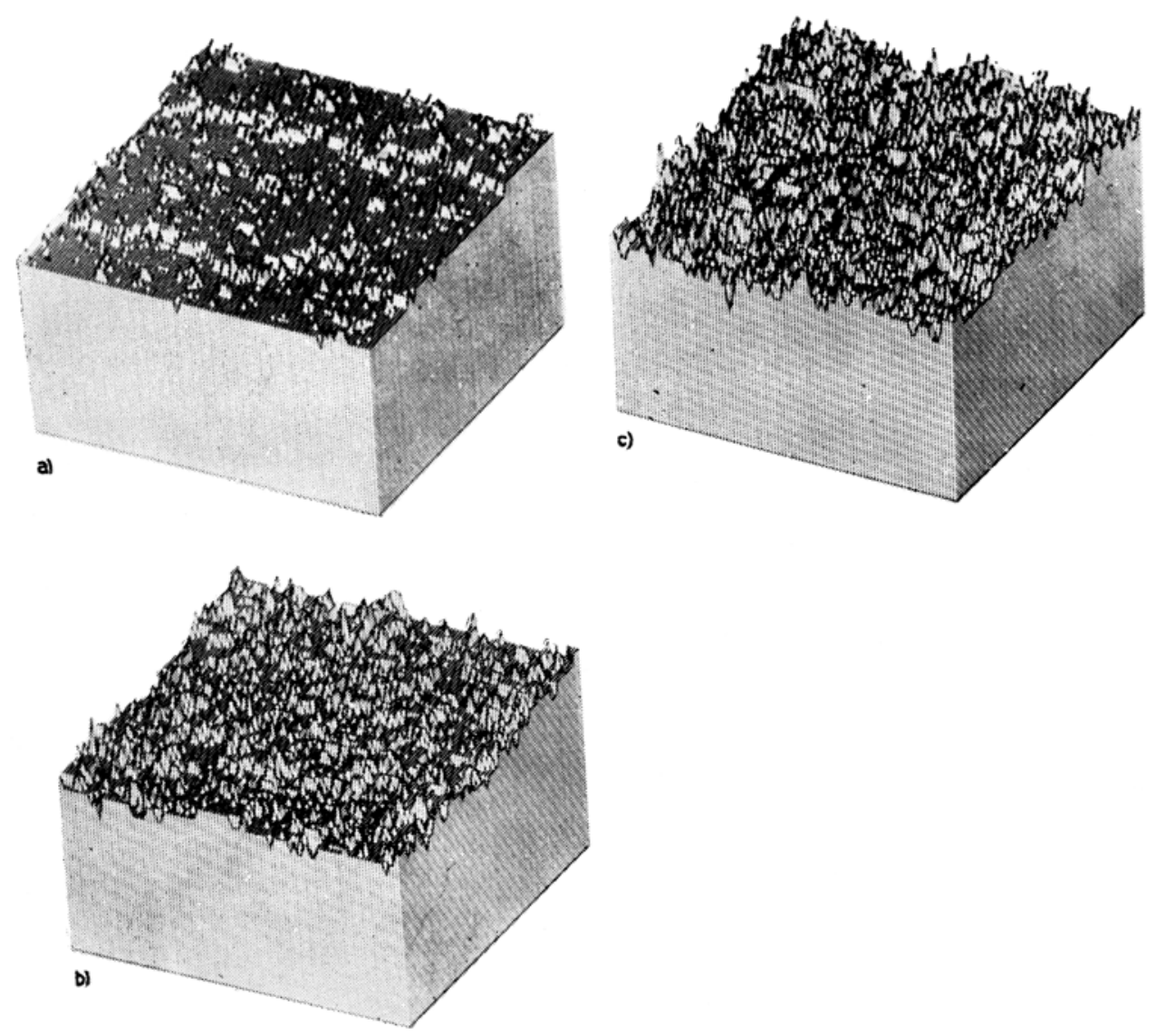

Higure 4. Monte Carlo simulation of a surface with steps at three different tenmeratures, ttamely, $T, T_{R}, T \sim T_{R}$ and $T, T_{R}$. (After Muller Krumbhaar 1979). 


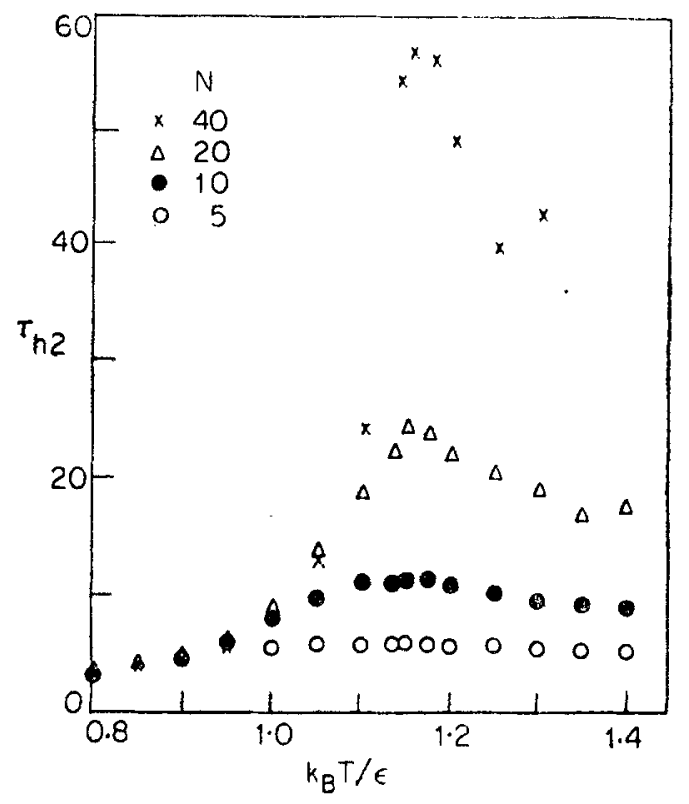

Figure 5. Relaxation time $\tau_{2}$ plotted versus temperature, $N$ refers to the edge length (after Swentsen 1976).

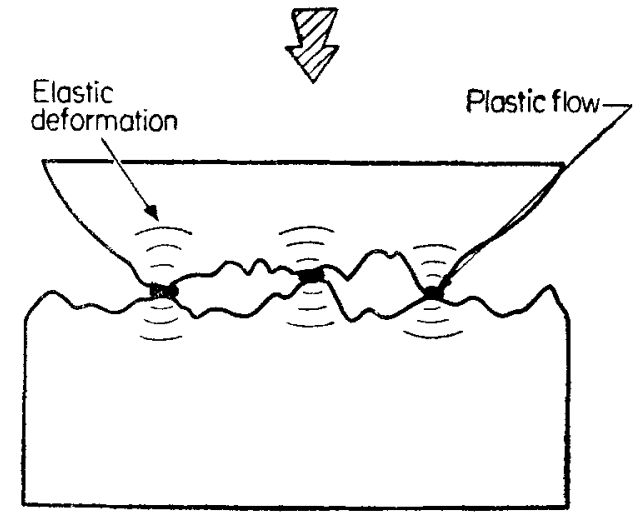

Figure 6. Schematic illustration of the contact between two rough surfaces. Contact occurs only near the high points where, on account of pressure magnification yielding could occur.

consequent weak adhesive forces, growth of adhesion cccurs due to the high deformability. By contrast, diamond will not adhere on contact, being very hard. One wonders whether this autocatalytic growth of adhesion occurs at a critical pressure and whether this bas features similar to a non-equilibrium phase transition. The question is well worth exploring. 


\section{Experiments on adhesion}

Many different kinds of experiments have been attempted to study various aspects of adhesion. As far as metals are concerned (and from now on we shall be mainly concerned with these and alloys), a well-developed technique due to Bowden (Bowden and Rowe 1956) involves first pressing two surfaces together and then 'tearing' them apart. Such studies have yielded valuable qualitative results. For instance, it has been found that if a shear is also applied while one surface is loaded on another, the force required later to pull apart the two surfaces increases. This increase is due to the enhanced adhesion promoted by plastic deformation caused by the shear. From the quantitative aspect, the results show much scatter.

Dynamic methods offer a possible means of overcoming some of the difficulties in static methods like the ones employed by Bowden. In particular, dissipation and surface forces can be allowed for separately, at least to first order, whereas in the static experiments, dissipative forces are difficult to take account of properly in the analysis.

One version of the dynamic method involves a sphere impacting a flat surface. Upcn impact the sphere rebounds and the coefficient of restituticn is measured hcre for various impact velocities until a critical velocity is reached below which adhesion occurs preventing restitution. It is obvious that gravity, entering through the weight of the sphere, could be a complicating factor which is why one seeks to carry out the experiment under microgravity conditions. The rest of this paper will be devoted to a description of one such experiment by Adorni et al (1979), proposed to be flown in the first Spacelab mission.

Figure 7 amplifies the principle of the experiment. On the to $p$ is sketched the repeated bounce of, say, a ping-pong ball. The experiment considered envisages a similar situation. Whereas a ping-pong ball could start from rest and acquire an initial velocity, we envisage the impacting sphere to be given a controlled initial velocity. Figure $7 \mathrm{~b}$ shows the velocity as a function of time. Not only are there many reversals, but also, on the average, the magnitude of the velocity decreases due to dissipation, with increasing number of bounces. A stage will clearly be reached when the energy restored to the ball will be too small to make it bounce upward, the ball being held down by both gravity and adhesion. To make the latter force dominate, we clearly have to go to a micrs gravity environment.

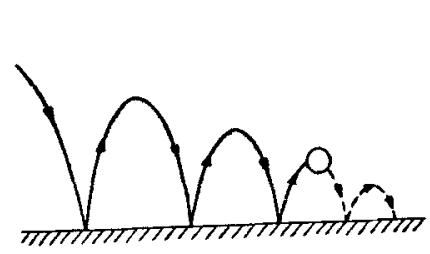

(a)

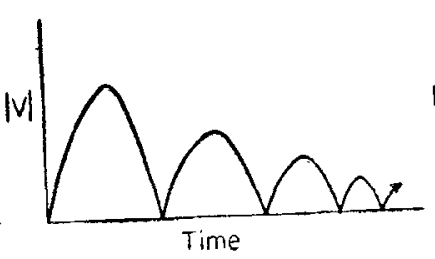

(b)

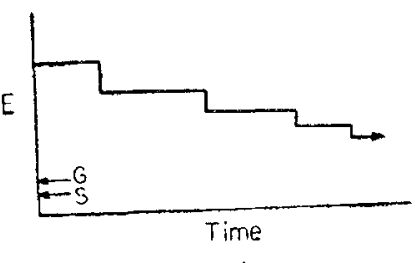

(c)

Figure 7. (a) Sketches the multiple bounces of a ping-pong ball. The magntitude of the velocity changes with time as in (b). The total entergy of the ball decreases after each bounce since it loses some energy every time. When the entergy restored to the sphere is less thin either the gravitational or adhesive pull, the sphere can nowlonger bounce up. The arrows $G$ and $S$ show schematically the gravitational and surface energies. 
To proceed further, we need to have some idea of what happens when one body impacts against another. The first worthwhile statement in this context appears to have been made by Newton who observed that impact can be divided into two categories, (i) perfectly elastic and (ii) imperfectly elastic, the latter being charac. terised by dissipation.

Quantitative studies on impact started with Hertz who confined himself to the first of the two categories mentioned above, which clearly rules out cases like impact by bullets, for example. Hertz prcceeds by considering first the elastic distorticn prcduced by impact. He assumes that the stresses set up are the same as those which would be produced if the load was turned on slowly. In modern parlance, we would call this the adiabatic apprcximation.

The next question one faces concerns the duration of the impact. To estimate this, we need to define a contact parameter $a$, which is done in figure 8a for two colliding spheres. $a$ is non zero during contact. A similar definition is available for a sphere impacting a slab. In figure $8 b$ is sketched the time variation of $a$. The related variation of $F$, the total force, can be obtained from

$$
F=-\partial U / \partial \alpha
$$

where $U$, the potential energy, is given by (Love 1944)

$$
U=\frac{2}{5 D}\left(\frac{r_{1} r_{2}}{r_{1}+r_{2}}\right) a^{5 / 2} \equiv \frac{1}{2} K a^{5 / 2},
$$

$D$ being defined as

$$
D=\frac{3}{4}\left(\frac{1-\sigma_{1}^{2}}{E_{1}}+\frac{1-\sigma_{2}^{2}}{E_{2}}\right)
$$

where the $\sigma$ 's denote Poisson ratios and the $E$ 's the Young's modulii of the materials of the two spheres respectively. From above we observe

$$
F \sim a^{3 / 2} .
$$

The kinetics of impact can be followed through via the equation of motion

$$
M \bar{a}=-\frac{5}{2} K a^{3 / 2}
$$

where $M=\left[m_{1} m_{2} /\left(m_{1}+m_{2}\right)\right]$ is the reduced mass.

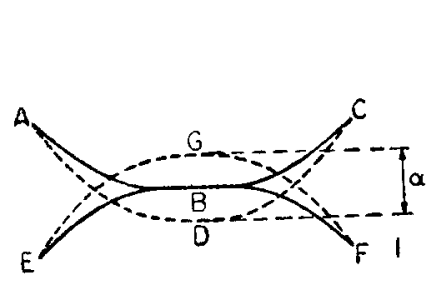

(o)

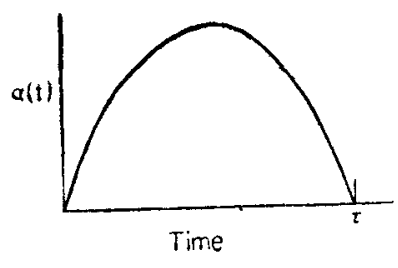

(b)

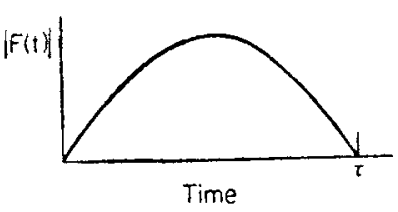

(c)

Figure 8. (a) Contact parameter $a$ for two colliding spheres. The dctted lines indicate the positions in the absence of elastic deformation. (b) shows the variation of $a$ with time while (c) sketches the variation of the niagnitude of the total force, 
The initial condition is $\bar{a}(0)=v_{1}(0)$, where $v_{1}(0)$ is the incident velocity, for convenience we assume that initially one sphere is at rest. Figure $8 \mathrm{c}$ shows how the force varies with time; the quantity $\tau$ defines the impact time.

The analysis sketched here can be extended to a sphere impacting a semiinfunite slab, along similar lines. In particular,

$$
\tau=2.94 Q / v_{1}(0)
$$

where $Q=\left[\frac{15}{16} v_{1}^{2}(0)\left(\frac{1-\sigma_{1}^{2}}{E_{1}}+\frac{1-\sigma_{2}^{2}}{E_{2}}\right) m_{1}\right]^{2 / 5} r_{1}^{-1 / 5}$

It is noteworthy that considerations of gravity have so far not entered into the analysis. We simply assume that the projectile has a velocity $v_{1}(0)$, not large enough to cause dissipation but high enough to outweigh (!) gravity.

Way back, Raman $(1918,1920)$ carried out some experiments to verify aspects of Hertz's theory. He performed two series of experiments, one involving spheres colliding with each other and another involving spheres falling on flat plates. In all these, the coefficient of restitution $e$ defined by

$$
e=-v_{1}(\tau) / v_{1}(0)
$$

was measured. Figure 9 gives typical results for colliding spheres (Raman 1918). The diameter of the sphere is not explicitly mentioned but from general statements made in the paper, it would appear to be $\sim 1 \mathrm{~cm}$.

In the case of spheres impacting on plates, Raman estimated $e$ from theory as follows: Invoking an earlier calculation by Lamb as to what happens to a plate when subject to an impulse loading at the origin, he argues that, when the sphere impacts, surface waves both symmetric and antisymmetric will be set up as in figure 10. Of the two, only the antisymmetric waves are expected to be dominant, and that too only the slowly moving ones. The radius $a$ over which the flexural wave would spread at the termination of impact is given by

$$
a=V \tau=\lambda / 2
$$

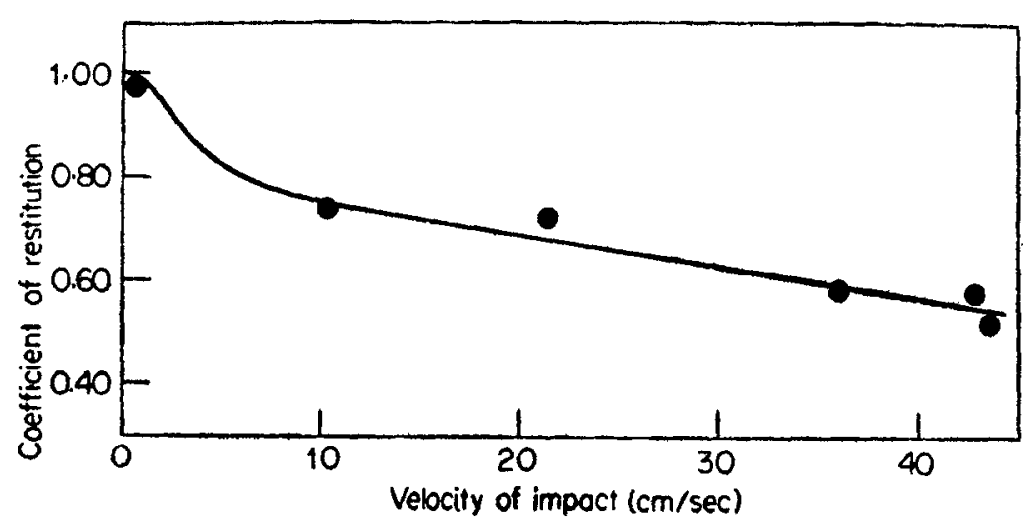

Figure 9. Coofficient of restitution for brass spheres as a function of impact velocity (After Raman 1918). 
where $\lambda$ is the wavelength and $V$ the group vel city. The latter is related to the material properties of the plate by

$$
V^{2}=\frac{4}{3} \pi \frac{f^{2}}{\lambda^{2}} \frac{E_{2}}{\rho_{2}\left(1-\sigma_{2}^{2}\right)},
$$

where $2 f$ is the thickness of the plate and $\rho_{\mathbf{a}}$ its density. Guided by Lamb, Raman then assumes a velocity profile as in figure 11. The kinetic energy of the wave is now calculated to be

$$
\mathscr{E}=\int_{0}^{0} \frac{1}{2} 2 f \rho_{2} 2 \pi r^{2} v(r) d r
$$

The total energy of the wave, which is the sum of its kinetic and potential energies is approximated as $2 \mathscr{E}$. The impulse given by the impinging body is

$$
I=\int_{0}^{a} 2 f \rho_{2} 2 \pi r v(r) d r
$$

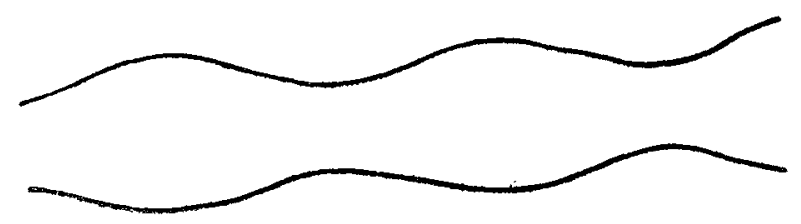

(a)

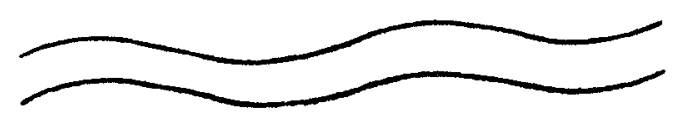

(b)

Figure 10. Lamb waves excited in a plate by impact; (a) the symmetric mode and (b) the antisymmetric mode.

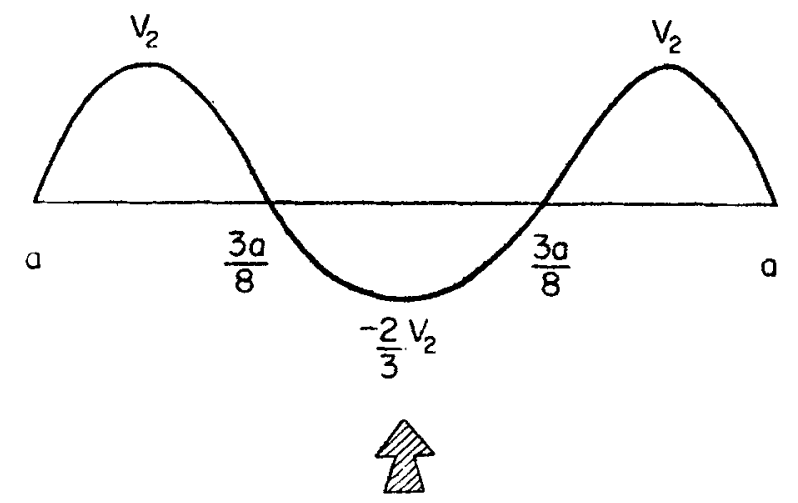

Figure 11. Velocity profile for the Lamb wave considered by Raman. The arrow shows the direction of impact. 
Conservation of energy and momentum then leads to

$$
\begin{aligned}
& \frac{1}{2} m_{1} v_{1}^{2}(0)\left(1-e^{2}\right)=2 \mathscr{E}, \\
& m_{1} v_{1}^{2}(0)(1+e)=I,
\end{aligned}
$$

from which $e$ is solved. Hertz's theory enters via the impact time $\tau$ (cf equations $(6-8))$. A factor of uncertainty in the analysis is the precise nature of the Lamb wave excited. The profile of figure 11 is only a guess, and as the thickness changes this profile could alter.

For the case of colliding spheres, Raman established that $e$ increases and approaches unity as the velocity of impact decreases (see figure 9). In the case of spheres impacting on plates, the experiments were done with an initial velocity of $234 \mathrm{~cm} / \mathrm{sec}$. The sphere diameter and the plate thickness were varied; $e$ was computed as described earlier and comparisons with experiment were made. The trends of the experimental data could be completely understood, and although results for impact on plates were not obtained at different velocities, it was clear a variation as in figure 9 was to be expected.

Despite the agreement between theory and experiment, it is clear that Raman's analysis cannot be extended to very small velocities on account of his neglect of surface and gravitational forces.

We now come to the experiment proposed by Adorni et al (1979). This specifically aims at measuring $\gamma$ by the rebound technique, i.e., by measuring the threshold impact velocity above which only rebound occurs; rebound does not occur for lower velocities due to adhesion.

Figure 12 gives an idea of the arrangement which is enclosed in a UHV chamber. The impacting sphere is made of stainless steel AISI 420 and has a diameter of $3.17 \mathrm{~mm}$. Till the experiment is actually started, the sphere is held in a special device employing four ruby spheres in a tetrahedral array enclosing the test sphere. Ruby is chosen to avoid possible soldering of the experimental sphere to the device during the long time foreseen from the delivery of the apparatus to the performance of the experiment in space. At the start of the experiment, the sphere is allowed to fall on to the target surface. The latter is made of stainless steel AISI 325 , and is linked to a piezoelectric transducer. The entire arrangement is in a magnetic field which simulates a microgravity level $\sim 10^{-2} \mathrm{~m} \mathrm{sec}-2$, which is to be compared with the $g$-jitter level $10^{-3} \mathrm{~m} \mathrm{sec}-2$ expected in the spacecraft. The bounce surface is arranged to have a slightly concave shape, with its symmetry axis coinciding with that of the magnetic field. This is expected to facilitate focussing the sphere rebound motion along a line of force near the symmetry axis of the field.

After the sphere drops on to the test surface, a percussion system is actuated by an electromagnet and an impulse is given to the base of the transducer. Its momentum is then transferred to the sphere causing it to jump about $1 \mathrm{~mm}$, starting thereby a sequence of bounces. The transducer serves to monitor the bounces, and data is acquired with an appropriate data acquisition system.

The experiment is yet to be performed. Data will be acquired for various percussional forces and various magnetic fields. Some feel for the kind of data likely to be generated is already available from computer simulation (see figure 13). Comparing with figure 9 , we see a different trend as the impact velocity decreases, 


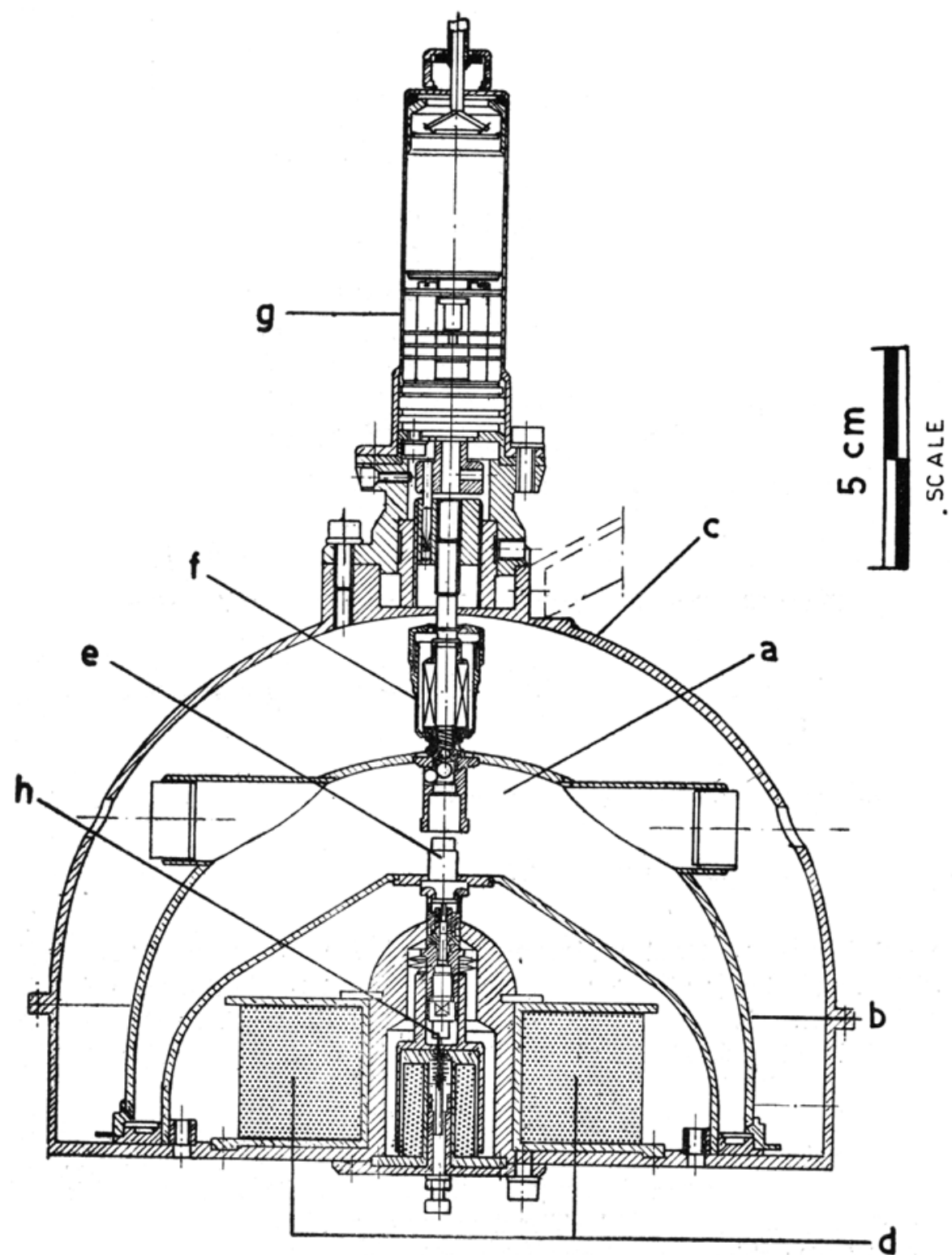

Figure 12. Main mechanical units of the UHV chamber. (a) UHV chamber, (b) connection to pump, (c) magnetic field bowl, (d) field coil (e) transducer, $(\mathfrak{f})$ sphere holding subsystem, (g) Sphere releasing subsystem, (h) percussion subsystem (after Adorni et al 1979).

However, there is no contradiction, and to appreciate this, let us consider the equation of motion as given by Adorni et al (1971) which is

$$
M \ddot{\alpha}=F_{C}+F_{S}+W .
$$




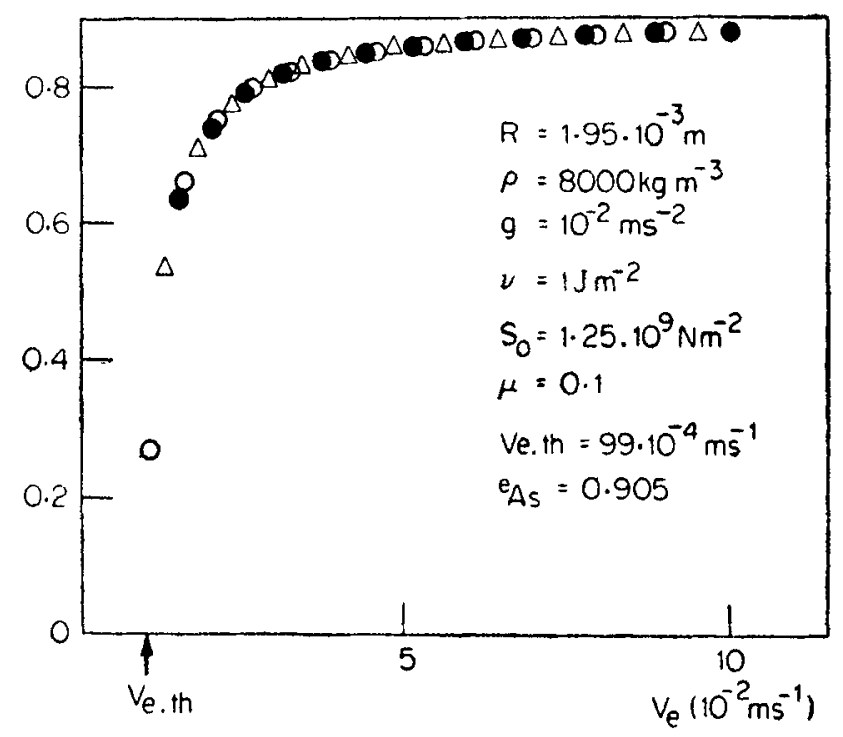

Figure 13. Computer simulation of impact results (after Adorni et al 1979).

Here $F_{C}$ is the contact force, $F_{S}$ the surface force and $W$ that due to gravity. $F_{c}$ is modelled as

$$
\begin{aligned}
F_{C} & =-(1+\mu) S_{0} R a & & 0<t<\frac{\tau}{2} \\
& =-(1-\mu) S_{0} R a & & \frac{\tau}{2}<t<\tau
\end{aligned}
$$

where $S_{0}$ is a parameter dependent on mechanical properties, $\mu$ is the coefficient of friction and $R=\left(r_{1} r_{2} /\left(r_{1}+r_{2}\right)\right)$ is the reduced radius of the two bodies in the contact region. The surface force is given by $F_{\mathrm{s}}=4 \pi R \gamma$. Besides friction, Raman ignored both $F_{S}$ and $W^{\dagger}$. Further, following Hertz, $F_{C}$ was modelled by him as $\sim a^{3 / 2}$ (see equation (3)).

For spheres impacting on plates, Raman did not measure $e$ as a function of decreasing velocity. Had he done so, he would have initially found $e$ increasing, as in figure 9. However, for very small velocities, the trend would have been different, being as in figure 14. The downward trend of $e$ as $v \rightarrow o$ is caused by $F_{s}$ and $W$. By going to a microgravity environment, Adorni et al seek to render negligible, the effect of the $W$ term. Under these conditions, the threshold velocity is calculated by them to be given by

$$
V_{\mathrm{th}}=\left(\frac{8 \mu F_{\mathrm{s}}}{M S_{0} R(1-\mu)}\right)^{1 / 2}
$$

which shows how $\gamma$ may be extracted.

t Fof the sphere on plate case, the following are the different energies (all in ergs). Kinetic energy of sphere just before impact $\sim 82134$; energy of the Lamb wave $\sim 3252$; gravitational energy $\sim 3 \cdot 8$, and surface energy $\sim 4 \cdot 7$. 


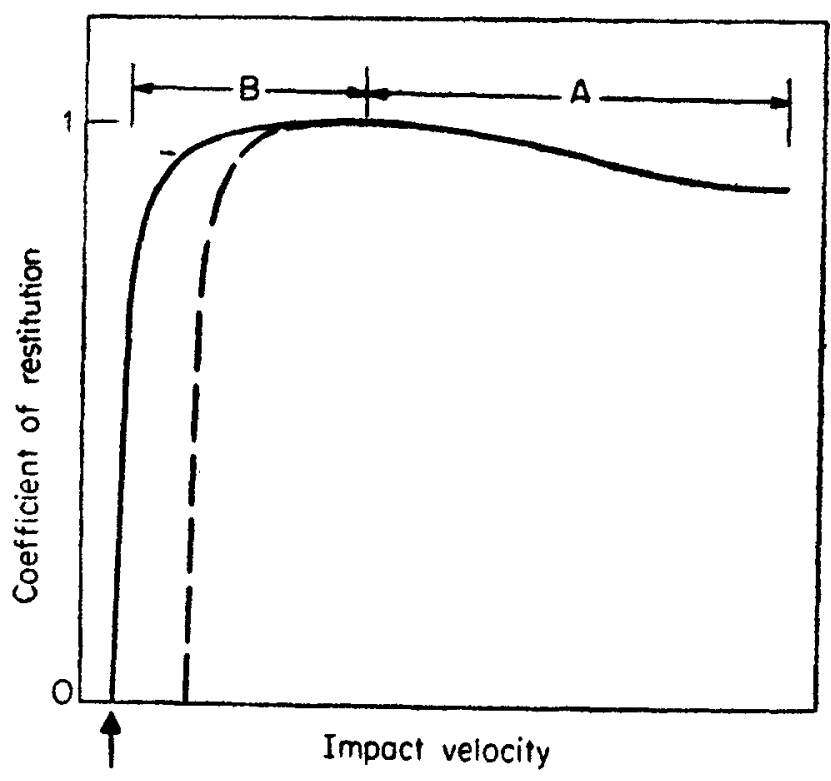

Figure 14. Schematic plot of $e$ versus impact velocity. Region $A$ is that explored by Raman in his experiments while region B is what is proposed to be covered in the Soacelab experiments. The arrow shows the threshold velocity by measuring which, surface energy is obtained. The dashed curve would result if gravitational energy is comparable to surface energy. In this case, the threshold velocity will not be determined by surface forces alone.

Now comes the question about the significance of the final result, when it is eventually obtained. Here one tends to have some reservations. From the basic physics point of view, stainless steel is hardly the best candidate to start with. From the practical angle also it is not clear how an accurate value of $\gamma$ for stainless steel obtained in UHV is of any importance. Most of the real-life surface will always have adsorbed atoms which will clearly alter the adhesion properties.

The author's personal view is that many experiments concerning adhesion need to be done and the state of the art is such that a lot more is yet to be done here on earth before we venture into space. Of course the inclination to design such experiments is generally missing. Possibly, experiments sucb as the one proposed by Adorni et al will provide sufficient challenge and provocation to come up with less expensive and more purposeful alternate experiments which can be conveniently done in terrestial laboratories.

\section{Acknowledgements}

The author would like to express his thanks to Dr D Sahoo for several helpful discussions concerning the impact experiments. Shri P Subba Rao assisted with the typing and Shri Ajit Kumar with the tracings. 


\section{References}

Adorni Net al 1979 Proc. II European symp. on materials in space, Grenoble ESA-SP-

Bowden F P and Rowe G W 1956 Proc. R. Soc. (London) A239 429

Gregg S J 1965 Surface chemistry of solids (London: Chapman and Hall)

Israelachivili and Tabor D 1972 Progress in surface and membrane science, (ed) J F Darielli (Academic Press) 5121

Love A E H 1944 A treatise on the mathematical theory of elasticity (New York: Dove,

Muller Krumblaar H 1979 IFF Bulletin No 13 Kernforschu 2 gzentrum Julich

Raman C V 1918 Phys. Rev. 12442

Raman C V 1920 Phys. Rev. 15277

Spiker I K and Barnes R 1970 Aerospace adhesives and elastomers (California : Society of Aerospace Materials and Process Engineer) 2

Swendsen R 1976 Phys. Rev. Lett. 371478 